\title{
On Weird and Pseudoperfect Numbers
}

\author{
By S. J. Benkoski and P. Erdös
}

\begin{abstract}
If $n$ is a positive integer and $\sigma(n)$ denotes the sum of the divisors of $n$, then $n$ is perfect if $\sigma(n)=2 n$, abundant if $\sigma(n) \geqq 2 n$ and deficient if $\sigma(n)<2 n$. $n$ is called pseudoperfect if $n$ is the sum of distinct proper divisors of $n$. If $n$ is abundant but not pseudoperfect, then $n$ is called weird. The smallest weird number is 70 .

We prove that the density of weird numbers is positive and discuss several related problems and results. A list of all weird numbers not exceeding $10^{6}$ is given.
\end{abstract}

Let $n$ be a positive integer. Denote by $\sigma(n)$ the sum of divisors of $n$. We call $n$ perfect if $\sigma(n)=2 n$, abundant if $\sigma(n) \geqq 2 n$ and deficient if $\sigma(n)<2 n$. We further define $n$ to be pseudoperfect if $n$ is the distinct sum of some of the proper divisors of $n$, e.g., $20=1+4+5+10$ is pseudoperfect [6]. An integer is called primitive abundant if it is abundant but all its proper divisors are deficient. It is primitive pseudoperfect if it is pseudoperfect but none of its proper divisors are pseudoperfect.

An integer $n$ is called weird if $n$ is abundant but not pseudoperfect. The smallest weird number is 70 and Table 1 is a list of all weird numbers not exceeding $10^{6}$. The study of weird numbers leads to surprising and unexpected difficulties. In particular, we could not decide whether there are any odd weird numbers [1] nor whether $\sigma(n) / n$ could be arbitrarily large for weird $n$. We give an outline of the proof that the density of weird numbers is positive and discuss several related problems. Some of the proofs are only sketched, especially, if they are similar to proofs which are already in the literature.

First, we consider the question of whether there are weird numbers $n$ for which $\sigma(n) / n$ can take on arbitrarily large values. Tentatively, we would like to suggest that the answer is negative. We can decide a few related questions. Let $n$ be an integer with $1=d_{1}<\cdots<d_{k}=n$ the divisors of $n$. We say that $n$ has property $\mathrm{P}$ if all the $2^{k}$ sums $\sum_{i=1}^{k} \epsilon_{i} d_{i}, \epsilon_{i}=0$ or 1 , are distinct. P. Erdös proved that the density of integers having property $\mathbf{P}$ exists and is positive [2]. Clearly, $2^{m}$ has property $\mathbf{P}$ for every $m$. It is plausible to conjecture that if $n$ has property $\mathrm{P}$, then $\sigma(n) / n<2$. The result is indeed true and follows from the next theorem. We conjectured this and the simple and ingenious proof is due to $C$. Ryavec.

THEOREM 1. Let $1 \leqq a_{1}<\cdots<a_{n}$ be a set of integers for which all the sums $\sum_{i=1}^{n} \epsilon_{i} a_{i}, \epsilon_{i}=0$ or 1 , are distinct. Then

$$
\sum_{i=1}^{n} \frac{1}{a_{i}}<2
$$

Proof. We have, for $0<x<1$,

Received June 28, 1973.

AMS (MOS) subject classifications (1970). Primary 10A40, 10J99, 10-04; Secondary 10H25. Key words and phrases. Weird numbers, pseudoperfect numbers, primitive abundant numbers. 
TABLE 1

Weird Numbers $\leqq 10^{\circ}$

\begin{tabular}{ll}
\hline Primitive & \multicolumn{1}{c}{ Nonprimitive } \\
\hline 70 & $70 \cdot p$ with $p>\sigma(70)$ and $p$ a prime \\
836 & $7192 \cdot 31$ \\
4030 & $836 \cdot 421$ \\
5830 & $836 \cdot 487$ \\
7192 & $836 \cdot 491$ \\
7912 & $836 \cdot p$ with $p \geqq 557$ and $p$ a prime \\
9272 & \\
10,792 & \\
17,272 & \\
45,356 & \\
73,616 & \\
83,312 & \\
91,388 & \\
113,072 & \\
243,892 & \\
254,012 & \\
338,572 & \\
343,876 & \\
388,076 & \\
519,712 & \\
539,774 & \\
555,616 & \\
682,592 & \\
786,208 & \\
\hline
\end{tabular}

$$
\prod_{i=1}^{n}\left(1+x^{a i}\right)<\sum_{k=0}^{\infty} x^{k}=\frac{1}{1-x}
$$

Thus

$$
\sum_{i=1}^{n} \log \left(1+x^{a_{i}}\right)<-\log (1-x) \text { or }
$$

$\sum_{i=1}^{n} \int_{0}^{1} \frac{\log \left(1+x^{a_{i}}\right)}{x} d x<-\int_{0}^{1} \frac{\log (1-x)}{x} d x$.

Now, putting $x^{a_{i}}=y$, we obtain, from (1),

$$
\sum_{i=1}^{n} \frac{1}{a_{i}} \int_{0}^{1} \frac{\log (1+y)}{y} d y<-\int_{0}^{1} \frac{\log (1-x)}{x} d x
$$

i.e., 


$$
\sum_{i=1}^{n} \frac{1}{a_{i}}\left(\frac{\pi^{2}}{12}\right)<\frac{\pi^{2}}{6}
$$

Thus $\sum_{i=1}^{n} 1 / a_{i}<2$ and the theorem is proved.

The same argument can be used to show that if the sums $\sum_{i=1}^{n} \epsilon_{i} a_{i}$ are all distinct, then

$$
\sum_{i=1}^{n} \frac{1}{a_{i}} \leqq 2-\frac{1}{2^{n-1}}
$$

and equality holds only if $a_{i}=2^{i-1}, i=1,2, \cdots, n$.

Here, we call attention to an old conjecture of $P$. Erdös. If the sums $\sum_{i=1}^{n} \epsilon_{i} a_{i}$, $\epsilon_{i}=0$ or 1 , are all distinct, then, is it true that $a_{n}>2^{n-c}$ for an absolute constant $C$ ? P. Erdös offered and still offers 300 dollars for a proof or disproof of this conjecture.

Consider next the property $\mathbf{P}^{\prime}$. An integer $n$ is said to have property $\mathbf{P}^{\prime}$ if no divisor of $n$ is the distinct sum of other divisors of $n$. Here again, we can prove that there is an absolute constant $C$ so that $\sigma(n) / n>C$ implies that $n$ cannot have property $\mathbf{P}^{\prime}$. This is immediate from the following old result of P. Erdös [3].

THEOREM 2. Let $a_{1}<a_{2}<\cdots$ be a finite or infinite sequence of integers no term of which is the distinct sum of other terms; then $\sum_{i} 1 / a_{i}<C$ where $C$ is an absolute constant.

Proof. In view of the fact that the proof appeared in Hungarian, we give the outline of the proof here.

Put $A(x)=\sum_{a_{i \leq x}} 1$. We split the positive integers into two classes. In the first class are the integers $n$ for which

$$
A\left(2^{n+1}\right)-A\left(2^{n}\right)<2^{n} / n^{2}
$$

Clearly, from (2),

$$
\sum^{\prime} \frac{1}{a_{i}}<\sum_{n=1}^{\infty} \frac{1}{n^{2}}<2
$$

where $\sum^{\prime}$ is over all $j$ such that $2^{n}<a_{i} \leqq 2^{n+1}$ for some $n$ in the first class.

Let $n_{1}<n_{2}<\cdots$ be the integers belonging to the second class, i.e.,

$$
\sum_{2^{n_{i}}<a_{i} \leq 2^{n_{j}+1}} 1 \geqq 2^{n_{j}} / n_{i}^{2} .
$$

Observe that the integers

$$
a_{1}+a_{2}+\cdots+a_{r}+a_{k} \text { with } 1 \leqq r<k
$$

are all distinct since if $a_{1}+\cdots+a_{r_{1}}+a_{k_{1}}=a_{1}+\cdots+a_{r_{2}}+a_{k_{2},} r_{2}>r_{1}$, then $a_{k_{1}}$ would be a distinct sum of other $a_{i}$ 's.

Now, put $n_{[i / 2]}=t$. Clearly,

$$
n_{i} \geqq t+[j / 2] .
$$

By (4),

$$
A\left(2^{t+1}\right)-A\left(2^{t}\right) \geqq 2^{t} / t^{2}>5 t^{2}>j^{2} \quad \text { for } j>100 .
$$

Let $1 \leqq a_{1}<\cdots<a_{i}$, be the first $j^{2}$ of the $a_{i}$ 's. By (7), $a_{i} \leqq 2^{i+1}$. Consider now the integers (5) for $1 \leqq r \leqq j^{2}, a_{r}<a_{k} \leqq 2^{n_{i+1}}$. By (7), $a_{r}<2^{t+1}$. Thus, by (6), the 
integers (5) are all less than

$$
2^{n_{i}+1}+j^{2} 2^{t+1}<2^{n_{i}+2} \text { for } j>100 .
$$

Now, observe that there are at least

$$
j^{2}\left(A\left(2^{n_{j}+1}\right)-j^{2}\right)
$$

integers of the form (5); they are all distinct and are all less than $2^{n_{i+2}}$. Thus, from (8), (6), and (7),

$$
A\left(2^{n_{i}+1}\right)<j^{2}+\frac{2^{n_{j}+2}}{j^{2}}<10 \frac{2^{n_{j}}}{j^{2}} \text { for } j>100
$$

Now, (10) and (3) immediately imply the uniform boundedness of $\sum_{i}^{\infty} 1 / a_{i}$. It is perhaps not quite easy to get the best possible value of $C$. It seems certain that $C<10$.

Unfortunately, we obtain no information about pseudoperfect numbers by these methods.

It is known that the density of integers having property $P$ exists and that the same holds for $\mathrm{P}^{\prime}$ (see [2]). Denote by $u_{1}<u_{2}<\cdots$, respectively $v_{1}<v_{2}<\cdots$, the integers which do not have property $\mathbf{P}$, respectively $\mathbf{P}^{\prime}$, but all of whose proper divisors have property $\mathrm{P}$, respectively $\mathrm{P}^{\prime}$. We expect that $\sum_{i} 1 / u_{i}$ and $\sum_{i} 1 / v_{i}$ both converge and, in fact, that

$$
\sum_{u_{i} \leq x} 1=O\left(\frac{x}{(\log x)^{k}}\right), \quad \sum_{i \leq x} 1=O\left(\frac{x}{(\log x)^{k}}\right)
$$

for every $k$ but have not been able to find a proof. For primitive abundant numbers, the analogous results and much more is true [4].

Now, consider weird and pseudoperfect numbers. Ań integer is primitive pseudoperfect if it is pseudoperfect but all its proper divisors are not pseudoperfect. It seems certain that the number of primitive pseudoperfect numbers not exceeding $x$ is $O\left(x /(\log x)^{k}\right)$ and, hence, the sum of their reciprocals converges. This we could not prove, but the fact that the density of the pseudoperfect numbers exists follows by the methods of [2]. It is easy to prove that there are infinitely many primitive abundant numbers which are pseudoperfect and, therefore, primitive pseudoperfect. The integers $2^{k} p$, with $p$ a prime such that $2^{k}<p<2^{k+1}$, are easily seen to be primitive abundant and pseudoperfect. In fact, they are practical numbers of Grinivasan, i.e., every $m \leqq \sigma\left(2^{k} p\right)$ is the distinct sum of divisors of $2^{k} p$. We leave the simple proof to the reader.

It is slightly less trivial to prove that there are infinitely many primitive abundant numbers all of whose prime factors are large and which are pseudoperfect. We only outline the proof.

For every $k$, let $f(k)$ be the smallest index for which $\left(p_{1}<p_{2}<\cdots\right.$ are the consecutive prime numbers) $\sigma\left(p_{k} \cdots p_{f(k)}\right) \geqq 2 p_{k} \cdots p_{f(k)}$.

THEOREM 3. There exists a positive integer $k_{0}$ such that, for $k>k_{0}$, the integers

$$
A_{k}=\prod_{k \leq l \leq f(k)} p_{l} \quad \text { and } \quad B_{k}=\left(A_{k} / p_{f(k)}\right) p_{f(k)+1} p_{f(k)+2}
$$

are both primitive pseudoperfect.

Note that $B_{1}=70$ which is not pseudoperfect. It appears that this is the only 
value of $k$ for which Theorem 3 fails, but to prove this might be difficult and would certainly require long computations for $B_{k}$ and perhaps a new idea for $A_{k}$.

We need two lemmas.

LEMMA 1. There is an absolute constant $c$ such that every integer $m>c p_{k}$ is the distinct sum of primes not less than $p_{k}$.

The lemma is probably well known and, in any case, easily follows by Brun's method.

LEMMA 2. There exists an integer $k_{0}$ such that, for every $k>k_{0}$,

$$
c p_{k}<m<\sigma\left(A_{k}\right)-c p_{k}
$$

implies that $m$ is the distinct sum of divisors of $A_{k}$. The same result holds for $B_{k}$.

Lemma 2 follows easily from Lemma 1 and from the fact that, for $p_{k} \leqq x$ $<\frac{3}{2} x<A_{k}$, the interval $\left(x, \frac{3}{2} x\right)$ always contains a divisor of $A_{k}$ and $B_{k}$. (To prove this last statement, we only need that, for $\epsilon>0$, there exists an integer $i_{0}(\epsilon)$ such that $p_{i+1}<(1+\epsilon) p_{i}$ for $i>i_{0}(\epsilon)$.)

Lemma 2 implies Theorem 3 if we can show

$$
\sigma\left(B_{k}\right)-2 B_{k}>c p_{k} \text { and } \sigma\left(A_{k}\right)-2 A_{k}>c p_{k} .
$$

Statement (12) follows immediately for $B_{k}$ by a very simple computation if we observe that there is an integer $l_{0}$ such that, for $l>l_{0}$,

$$
\left(1+1 / p_{l+1}\right)\left(1+1 / p_{l+2}\right)>1+3 / 2 p_{l} .
$$

We do not have such a simple proof of (12) for $A_{k}$. Observe that

$$
\sigma\left(A_{k}\right)-2 A_{k} \geqq\left(\sigma\left(A_{k}\right), A_{k}\right)
$$

where $(a, b)$ denotes the greatest common divisor of $a$ and $b$.

Now, (13) implies (12) if we can show that $\left(\sigma\left(A_{k}\right), A_{k}\right)$ has, for $k>k_{0}$, at least two prime factors. In fact, we shall prove that $(\omega(n)$ denotes the number of prime factors of $n$ )

$$
\lim _{k \rightarrow \infty} \omega\left(\left(\sigma\left(A_{k}\right), A_{k}\right)\right)=\infty .
$$

To prove (14), we first observe that, for $\epsilon>0$, there is an integer $k_{0}(\epsilon)$ such that, for $k>k_{0}(\epsilon)$,

$$
p_{f(k)}>p_{k}^{2-\bullet} \text {. }
$$

This is of course well known and follows from the theorems of Mertens. The following theorem now implies (14).

THEOREM 4. Denote by $g(x)$ the number of indices $l_{1}$ for which there is an $l_{2}$ satisfying

$$
x<p_{l_{1}}<p_{l_{2}}<x^{2}, \quad p_{l_{2}} \equiv-1\left(\bmod p_{l_{1}}\right) .
$$

We then have $\lim _{x \rightarrow \infty} g(x)=\infty$.

Theorem 4 follows easily from the proof of Motohashi's theorem [5]. It does not follow from the theorem of Motohashi but it is easy to deduce by the same proof. Motohashi uses some deep results of Bombieri. Thus, (14) and Theorem 3 are proved.

It seems likely that there are infinitely many primitive abundant numbers which 
are weird but this we cannot prove. We can, however, show that the density of weird numbers is positive.

It is clear that the weird numbers have a density since both the abundant numbers and the pseudoperfect numbers have a density. (A weird number is abundant and not pseudoperfect.) Hence, we need only show that the density of weird numbers cannot be 0 . This follows from the following simple lemma.

LemMa. If $n$ is weird, then there is an $\epsilon_{n}>0$ such that $n t$ is weird if

$$
\sum_{d \mid t} \frac{1}{d}<1+\epsilon_{n} .
$$

Proof. First define $(x)^{+}$by

$$
\begin{aligned}
(x)^{+}=x & \text { if } x \geqq 0, \\
& =\infty \quad \text { if } x<0 .
\end{aligned}
$$

Put

$$
\delta_{n}=\min \left(1-\sum^{\prime} 1 / d\right)^{+}
$$

where, in $\sum^{\prime}, d$ runs over all subsets of the divisors greater than 1 of $n$. If $n$ is deficient or weird, then $\delta_{n}>0$.

If $n t$ is not weird, then there is a set of divisors, greater than 1, of $n t$ for which

$$
1=\sum_{1} 1 / d+\sum_{2} 1 / d
$$

where, in $\sum_{1}, d \mid n$ and, in $\sum_{2}, d \mid n t$ but $d \nmid n$.

From (16) and (17),

$$
\frac{\sigma(t)}{t} \frac{\sigma(n)}{n} \geqq \sum_{2} \frac{1}{d} \geqq \delta_{n},
$$

which proves the lemma for $\epsilon_{n}=n \delta_{n} / \sigma(n)$.

THEOREM 5. The density of weird numbers is positive.

Proof. If $n$ is weird, then let $\epsilon_{n}$ be as in the proof of the lemma. Now, by the lemma, if $t$ is an integer and $\sigma(t) / t<1+\epsilon_{n}$, then $n t$ is weird. But the density of the integers $t$ with $\sigma(t) / t<1+\epsilon_{n}$ is positive for any $\epsilon_{n}>0$.

Actually, we proved a slightly stronger result. If $n$ is weird, then the density of $\{m ; n \mid m$ and $m$ is weird $\}$ is positive.

It is easy to see that if $n$ is weird and $p$ is a prime greater than $\sigma(n)$, then $p n$ is also weird. More generally, the following result holds. Let $n$ be an integer which is not pseudoperfect, i.e., $n$ is deficient or weird. The integer $p n$ is pseudoperfect if and only if there is a set $A$ of proper divisors of $n$ and a set $B$ of divisors of $n$ where no $b \in B$ is a multiple of $p$, such that

$$
p\left(n-\sum_{d \in A} a\right)=\sum_{b \in B} b .
$$

We leave the simple proof to the reader.

Finally, we state without proof the following result: Let $t \geqq 0$ be an integer. The density of integers $n$ for which $n+t$ is the distinct sum of proper divisors of $n$ is positive. On the other hand, the density of the integers $n$, for which $n-t(t>0)$ is the sum of distinct divisors of $n$, is 0 . 
Department of Mathematics Pennsylvania State University

University Park, Pennsylvania 16802 and

Daniel H. Wagner, Associates

Station Square One

Paoli, Pennsylvania 19301

Mathematics Institute

Hungarian Academy

Budapest, Hungary

1. S. J. Benkoski, "Elementary problem and solution E2308," Amer. Math. Monthly, v. 79,1972 , p. 774 .

2. P. ERDös, "Some extremal problems in combinatorial number theory," Mathematical Essays Dedicated to A. J. Macintyre, Ohio Univ. Press, Athens, Ohio, 1970, pp. 123-133. MR 43 \# 1942.

3. P. ERDös, "Remarks on number theory. III," Mat. Lapok, v. 13, 1962, pp. 28-38. (Hungarian) MR 26 \#2412.

4. P. ERDös, "On primitive abundant numbers," J. London Math. Soc., v. 10, 1935, pp. 49-58.

5. Y. Мотонаsн, "A note on the least prime in an arithmetic progression with a prime difference," Acta Arith., v. 17, 1970, pp. 283-285. MR 42 \#3030.

6. W. SIERPINSKI, "Sur les nombres pseudoparfaits," Mat. Vesnik, v. 2(17), 1965, pp. 212-213. MR 33 \#7296.

7. A. \& E. ZACHARIOU, "Perfect, semiperfect and Ore numbers," Bull. Soc. Math. Grèce, v. 13. 1972, pp. 12-22. 